\title{
On completely generalized co-quasi-variational inequalities
}

\author{
Syed Shakaib Irfan \\ College of Engineering, P.O. Box 6677, Qassim University, Buraidah-51452, Al-Qassim, Kingdom of Saudi Arabia
}

\section{Email address:}

shakaib@qec.edu.sa (S. S. Irfan)

\section{To cite this article:}

Syed Shakaib Irfan. On Completely Generalized Co-Quasi-Variational Inequalities, Applied and Computational Mathematics. Vol. 2, No. 1, 2013, pp. 14-18. doi: 10.11648/j.acm.20130201.12

\begin{abstract}
In the present work, we introduce and study completely generalized quasi-variational inequality problem for fuzzy mappings. By using the definitions of strongly accretive and retraction mappings, we propose an iterative algorithm for computing the approximate solutions of this class of variational inequalities. We prove that approximate solutions obtained by the proposed algorithm converge to the exact solutions of completely generalized quasi-variational inequality problem.
\end{abstract}

Keywords: Completely Generalized Quasi-Variational Inequality, $m$-Accretive Mappings, Strongly Accretive, Retraction Mappings, Uniformly Smooth Banach Spaces, Convergence Analysis

\section{Introduction}

In 1989, Chang and Zhu [8] firstly introduced the concept of variational inequalities for fuzzy mappings in locally convex Hausdorff topological vector spaces and investigated existence theorems for some kinds of variational inequalities for fuzzy mappings. In recent years, it has been generalized and extended to many different directions, see for example $[1,2,4,6-8,10,11,13,14]$ and references therein.

Several kinds of variational and quasi-variational inequalities for fuzzy mappings are considered and studied by Chang [6], Chang and Huang [7], Noor [13] and Lee et al [11]. Motivated and inspired by the work going in this field, in this paper, we considered the completely generalized quasi-variational inequality problem in the setting of $\mathrm{Ba}-$ nach spaces. A new iterative algorithm for computing the approximate solutions of completely generalized quasivariational inequality problem is suggested. We also discuss the existence and convergence of iterative sequences generated by our algorithm.

\section{Formulations and Preliminaries}

Let $B$ be a real Banach space and $B^{*}$ be its topological dual space. Let $<.$, . $>$ be the dual pairing between $B$ and $B^{*}$ and $C B(B)$ be the family of all nonempty closed and bounded subset of $B$. Let $F(B)$ be a collection of all fuzzy sets over $B$. A mapping $P: B \rightarrow F(B)$ is said to be a fuzzy mapping. For each $x \in B, P(x)$ (denoted by $P_{x}$ in the sequel) is a fuzzy set on $B$ and $P_{x}(y)$ is the membership function of $y$ in $P_{x}$.

A fuzzy mapping $P: B \rightarrow F(B)$ is said to be closed if for each $x \in B$ the function $y \mapsto P_{x}(y)$ is upper semi continuous, that is, for any given net $\left\{y_{\alpha}\right\} \subset B$ satisfying $y_{\alpha} \rightarrow y_{0} \in B, \limsup _{\alpha} P_{x}\left(y_{\alpha}\right) \leq P_{x}\left(y_{0}\right)$. For $C \in F(B)$ and $\lambda \in[0,1]$ the set $(C)_{\lambda}=\{x \in B: C(x) \geq \lambda\}$ is called a $\lambda$-cut set of $c$.

A closed fuzzy mapping $A: B \rightarrow F(B)$ is said to satisfy condition $(*):$ if there exists a function $a: B \rightarrow[0,1]$ such that for each $x \in B,\left(A_{x}\right)_{a(x)}$ is a nonempty and bounded subset of $B$.

It is clear that if $A$ is a closed fuzzy mapping satisfying (*), then for each $x \in B$, the $\operatorname{set}\left(A_{x}\right)_{a(x)} \in C B(B)$.

In fact, $\left\{y_{\alpha}\right\}_{\alpha \in \Gamma} \subset\left(A_{x}\right)_{a(x)}$ be a net and $y_{\alpha} \rightarrow y_{0} \in B$. Then $A_{x}\left(y_{\alpha}\right) \geq a(x)$ for each $\alpha \in \Gamma$. Since $A$ is closed, we have

$$
A_{x}\left(y_{0}\right) \geq \limsup \operatorname{se\Gamma }_{\alpha \in} A_{x}\left(y_{\alpha}\right) \geq a(x) .
$$

This implies that $y_{0} \in\left(A_{x}\right)_{a(x)}$ and so $\left(A_{x}\right)_{a(x)} \in C B(B)$.

Let $P, Q, R, S: B \rightarrow F(B)$ be closed fuzzy mappings satisfying condition $(*)$. Then there exists functions $a, b, c, d: B \rightarrow[0,1]$ corresponding to $P, Q, R$ and $S$ respectively such that for each $x \in B$, we have 
$\left(P_{x}\right)_{a(x)},\left(Q_{x}\right)_{b(x)},\left(R_{x}\right)_{c(x)},\left(S_{x}\right)_{d(x)} \in C B(B)$. Therefore, we can define multi-valued mappings $\tilde{P}, \tilde{Q}, \tilde{R}, \widetilde{S}: B \rightarrow C B(B)$ by

$$
\begin{aligned}
& \tilde{P}(x)=\left(P_{x}\right)_{a(x)}, \\
& \tilde{Q}(x)=\left(Q_{x}\right)_{b(x)}, \tilde{R}(x)=\left(R_{x}\right)_{c(x)}, \tilde{S}(x)=\left(S_{x}\right)_{d(x)} \forall x \in B . \text { In the se- }
\end{aligned}
$$

quel, $\tilde{P}, \tilde{Q}$ and $\tilde{R}$ are called the multi-valued mappings induced by the fuzzy mappings $P, Q, R$ and $S$ respectively.

Let $T, A, H, N, G: B \rightarrow B$ be single valued mappings, $P, Q, R, S: B \rightarrow F(B)$ be fuzzy mappings. Let $a, b, c, d: B \rightarrow[0,1]$ be given functions. Let $K: B \rightarrow 2^{B}$ such that $\forall x \in B, K(x)$ is a nonempty, closed and convex. We consider the following completely generalized multi-valued quasi-variational inequality problem(CGQVIP)

Find $x, u, v$ and $w \in \operatorname{Bsuch}$ tha $\mathbb{P}_{x}(u) \geq a(x)$,

$$
\begin{aligned}
& \mathrm{Q}_{x}(v) \geq b(x), \mathrm{R}_{x}(w) \geq c(x), \mathrm{S}_{x}(r) \geq d(x), G(x) \in K(x) \text { and } \\
& \langle\mathrm{T}(u)+\mathrm{A}(v)+\mathrm{M}(w)+\mathrm{N}(\mathrm{r}), \mathrm{J}(\mathrm{z}-\mathrm{G}(\mathrm{x}))\rangle \geq 0 \forall z \in K(x), \mathrm{w}
\end{aligned}
$$
here $J: B \rightarrow B^{*}$ is the normalized duality mapping defined by

$$
\|J(x)\|_{*}=\|x\| \text { and }\langle x, J(x)\rangle=\|x\|^{2}, \forall x \in B .
$$

For further detail of the duality mapping $J$, we refer to [4].

We recall that uniform convexity of the Banach space $B$ means that for any given $\varepsilon>0$ there exists $\delta>0$ such that $\forall x, y \in B,\|x\| \leq 1,\|y\| \leq 1,\|x-y\|=\varepsilon$ ensure the following inequality

$$
\|x+y\| \leq 2(1-\delta)
$$

The function

$$
\delta_{B}(\varepsilon)=\inf \left\{1-\frac{\|x+y\|}{2}:\|x\|=1,\|y\|=1,\|x-y\|=\varepsilon\right\}
$$

is called the modulus of the convexity of the space $B$.

The uniform smoothness of the space $B$ means that for any given $\varepsilon>0$, there exists $\delta>0$ such that

$$
\frac{\|x+y\|+\|x-y\|}{2}-1 \leq \varepsilon\|y\|
$$

holds. The function

$$
\rho_{B}(t)=\sup \left\{\frac{\|x+y\|+\|x-y\|}{2}-1:\|x\|=1,\|y\|=t\right\}
$$

is called the modulus of the smoothness of space $B$.

Remark 2.1: The space $B$ is uniformly convex if and only if $\delta_{B}(\varepsilon)>0, \forall \varepsilon>0$, and it is uniformly smooth if and only if $\lim _{t \rightarrow 0} t^{-1} \rho_{B}(t)=0$.

The following inequalities will be used in the proof of our main result.

Proposition 2.1[3]: Let $B$ be a uniformly smooth Banach space and $J$ be a normalized duality mapping from $B$ to $B^{*}$, Then $\forall x, y \in B$, we have

$$
\begin{gathered}
\left\|x+y^{2}\right\| \leq\|x\|^{2}+2\langle y, J(x, y)\rangle, \\
\langle x-y, J(x)-J(y)\rangle \leq 2 d^{2} \rho_{B}\left(\frac{4\|x-y\|}{d}\right),
\end{gathered}
$$

Where

$$
d=\sqrt{\frac{\|x\|^{2}+\|y\|^{2}}{2}} .
$$

We next recall the following definitions.

Definition 2.1: A mapping $G ; B \rightarrow B$ is said to be Lipschitz continuous, if there exists a constant $\delta>0$ such that

$$
\|G(x)-G(y)\| \leq \delta\|x-y\|, \quad \forall x, y \in B .
$$

Definition 2.2: A set-valued mapping $P: B \rightarrow C B(B)$ is said to be $\widetilde{H}$-Lipschitz continuous, if there exists a constant $\beta>0$ such that

$$
\widetilde{H}(P(x), P(y)) \leq \beta\|x-y\|, \quad \forall x, y \in B .
$$

Where $\widetilde{H}(.,$.$) is the Housdorff metric on C B(B)$ defined by

$$
\begin{gathered}
\tilde{H}(X, Y)=\max \left\{\sup _{x \in X} d(x, Y), \sup _{y \in Y} d(X, y)\right. \\
\forall X, Y \in C B(B)
\end{gathered}
$$

Definition 2.3: The mapping $G: B \rightarrow B$ is said to be strongly accretive, if there exist a constant $\gamma>0$ such that

$$
\langle G(x)-G(y), J(x-y)\rangle \geq \gamma\|x-y\|^{2}, \forall x, y \in B .
$$

Definition 2.4[5,9]: A mapping $Q_{\Omega}: B \rightarrow \Omega$ where $\Omega$ be a nonempty, closed and convex subset of $B$ is said to be

(i) retraction on $\Omega$ if $Q_{\Omega}{ }^{2}=Q_{\Omega}$;

(ii) nonexpansive retraction if it satisfies the inequality

$$
\left\|Q_{\Omega} x-Q_{\Omega} y\right\| \leq\|x-y ;\| \forall x, y \in B ;
$$

(iii) sunny retraction if for all $x \in B$ and for all $-\infty<t<\infty$

$$
Q_{\Omega}\left(Q_{\Omega} x+t\left(x-Q_{\Omega} x\right)\right)=Q_{\Omega} x .
$$

Preposition 2.2[9]: $Q_{\Omega}$ is a sunny nonexpansive retraction if and only if $\forall x \in B$ and $\forall y \in \Omega$

$$
\left\langle x-Q_{\Omega} x, J\left(Q_{\Omega} x-y\right)\right\rangle \geq 0 .
$$

From the proposition 2.2, we have the following retraction shift inequality.

Preposition 2.3[4]: Let $\Omega$ be a nonempty, closed and convex subset of a Banach space $B$ and let $m: B \rightarrow B$ be a mapping. Then $\forall x \in B$, we have 


$$
Q_{\Omega+m(x)}=m(x)+Q_{\Omega}(x-m(x)) .
$$

\section{Existence and Convergence Results}

In this section, we construct the algorithm for finding approximate solutions for (CGQVIP) and the convergence of iterative sequences generated by algorithm.

We first recall the following results.

Lemma 3.1: Let $B$ be a real Banach space,

$T, A, M, N, G: B \rightarrow B$ be single valued mappings

$\widetilde{P}, \widetilde{Q}, \widetilde{R}, \widetilde{S}: B \rightarrow C B(B)$ and $K: B \rightarrow 2^{B}$ be multi-valued mappings such that $\forall x \in B, K(x)$ is nonempty closed and convex. Then the following statements are equivalent:

(a) The set of elements $x \in B, u \in \widetilde{P}(x), v \in \widetilde{Q}(x), w \in \widetilde{R}(x)$ and $r \in \widetilde{S}(x)$ is a solution of (CGQVIP).

(b) $x \in B, u \in \widetilde{P}(x), v \in \widetilde{Q}(x), w \in \widetilde{R}(x), r \in \widetilde{S}(x)$ and for any $\tau>0$

$$
G(x)=Q_{K(x)}[G(x)-\tau(T(u)+A(v)+M(w) N(r))] .
$$

Proof: It is similar to the proof of Theorem 1 in [4].

Combining Proposition 2.3 and Lemma 3.1, we have the following results.

Lemma 3.2: Let $B$ be a real Banach space and $X$ be a nonempty, closed and convex subset of $B$. Let $T, A, M, N G, m: B \rightarrow B$ be single valued mappings $\widetilde{P}, \widetilde{Q}, \widetilde{R}, \widetilde{S}: B \rightarrow C B(B)$ and $K: B \rightarrow 2^{B}$ be multi-valued mappings such that $\forall x \in B, K(x)=m(x)+X$. Then the set of elements $x \in B, u \in \widetilde{P}(x), v \in \widetilde{Q}(x) w \in \widetilde{R}(x)$ and $r \in \widetilde{S}(x)$ is a solution of (CGQVIP) if and only if

$$
\begin{gathered}
x=x-G(x)+m(x)+Q_{X}[G(x)-\tau(T(u)+A(v) \\
+M(w)+N(r))-m(x)],
\end{gathered}
$$

for any

$$
\tau>0
$$

To compute the approximate solution of (CGQVIP), we propose the following iterative algorithm.

Algorithm 3.1: Let $K(x)=m(x)+X$, where $X$ is a nonempty closed and convex subset of $B$ and $\tau>0$ be fixed. Let $P, Q, R, S: B \rightarrow F(B)$ be closed fuzzy mappings satisfying condition (*) and $\widetilde{P}, \widetilde{Q}, \widetilde{R}, \widetilde{S}: B \rightarrow C B(B)$ be multi-valued mappings induced by the fuzzy mappings $P, Q, R, S$ respectively. For given

$$
x_{0} \in B, u_{0} \in \widetilde{P}\left(x_{0}\right), v_{0} \in \widetilde{Q}\left(x_{0}\right) w_{0} \in \widetilde{R}\left(x_{0}\right),
$$

And

$$
r_{0} \in \widetilde{S}\left(x_{0}\right),
$$

let

$$
\begin{gathered}
x_{1}=x_{0}-G\left(x_{0}\right)+m\left(x_{0}\right)+Q_{X}\left[G\left(x_{0}\right)-\tau\left(T\left(u_{0}\right)+A\left(v_{0}\right)\right.\right. \\
\left.\left.+M\left(w_{0}\right)+N\left(r_{0}\right)\right)-m\left(x_{0}\right)\right] .
\end{gathered}
$$

By Nadler [12], there exists

$$
u_{1} \in \widetilde{P}\left(x_{1}\right), v_{1} \in \widetilde{Q}\left(x_{1}\right), w_{1} \in \widetilde{R}\left(x_{1}\right)
$$

and $r_{1} \in \widetilde{S}\left(x_{1}\right)$, such that

$$
\begin{aligned}
\left\|u_{0}-u_{1}\right\| & \leq \widetilde{H}\left(\widetilde{P}\left(x_{0}\right), \widetilde{P}\left(x_{1}\right)\right) ; \\
\left\|v_{0}-v_{1}\right\| & \leq \widetilde{H}\left(\widetilde{Q}\left(x_{0}\right), \widetilde{Q}\left(x_{1}\right)\right) ; \\
\left\|w_{0}-w_{1}\right\| & \leq \widetilde{H}\left(\widetilde{R}\left(x_{0}\right), \widetilde{R}\left(x_{1}\right)\right) ; \\
\left\|r_{0}-r_{1}\right\| & \leq \widetilde{H}\left(\widetilde{S}\left(x_{0}\right), \widetilde{S}\left(x_{1}\right)\right) .
\end{aligned}
$$

Let

$$
\begin{gathered}
x_{2}=x_{1}-G\left(x_{1}\right)+m\left(x_{1}\right)+Q_{X}\left[G\left(x_{1}\right)-\tau\left(T\left(u_{1}\right)\right.\right. \\
\left.\left.A\left(v_{1}\right)+M\left(w_{1}\right)+N\left(r_{1}\right)\right)-m\left(x_{1}\right)\right] .
\end{gathered}
$$

By induction, we can obtain sequences $\left\{x_{n}\right\},\left\{u_{n}\right\},\left\{v_{n}\right\},\left\{w_{n}\right\}$ and $\left\{r_{n}\right\}$ satisfying

$$
\begin{gathered}
u_{n} \in \widetilde{P}\left(x_{n}\right),\left\|u_{n}-u_{n+1}\right\| \leq \widetilde{H}\left(\widetilde{P}\left(x_{n}\right), \widetilde{P}\left(x_{n+1}\right)\right) ; \\
v_{n} \in \widetilde{Q}\left(x_{n}\right),\left\|v_{n}-v_{n+1}\right\| \leq \widetilde{H}\left(\widetilde{Q}\left(x_{n}\right), \widetilde{Q}\left(x_{n+1}\right)\right) ; \\
w_{n} \in \widetilde{R}\left(x_{n}\right),\left\|w_{n}-w_{n+1}\right\| \leq \widetilde{H}\left(\widetilde{R}\left(x_{n}\right), \widetilde{R}\left(x_{n+1}\right)\right) ; \\
r_{n} \in \widetilde{S}\left(x_{n}\right),\left\|r_{n}-r_{n+1}\right\| \leq \widetilde{H}\left(\widetilde{S}\left(x_{n}\right), \widetilde{S}\left(x_{n+1}\right)\right)
\end{gathered}
$$

and

$$
\begin{aligned}
x_{n+1}= & x_{n}-G\left(x_{n}\right)+m\left(x_{n}\right)+Q_{X}\left[G\left(x_{n}\right)-\tau\left(T\left(u_{n}\right)\right.\right. \\
& \left.\left.+A\left(v_{n}\right)+M\left(w_{n}\right)+N\left(r_{n}\right)\right)-m\left(x_{n}\right)\right] .
\end{aligned}
$$

We have the following existence and convergence result. Theorem 3.1. Let $B$ be a real uniformly smooth Banach space with the module of smoothness $\tau_{B}(t) \leq D t^{2}$ for some $D>0$. Let $X$ be a nonempty, closed and convex subset of $B$. Let $P, Q, R, S: B \rightarrow F(B)$ be closed fuzzy mappings satisfying condition $\left(^{*}\right.$ ) and $\widetilde{P}, \widetilde{Q}, \widetilde{R}, \widetilde{S}: B \rightarrow C B(B)$ be multivalued mappings induced by the fuzzy mappings $P, Q, R, S$ respectively. Let $\widetilde{P}, \widetilde{Q}, \widetilde{R}$ and $\widetilde{S}$ be $\widetilde{H}$-Lipschitz continuous mappings with constants $\beta, \eta, \psi$ and $\sigma$, respectively. Let $T, A, M, N, G, m: B \rightarrow B$ be Lipschitz continuous with constant $\alpha, \lambda, \theta, \varphi$ and $\chi$ respectively, $G: B \rightarrow B$ be both strongly accretive with constant $\gamma>0$ and Lipschitz continuous with constant $\delta>0$. If

$$
\begin{aligned}
& 0<\left(1-2 \gamma+64 D \delta^{2}\right)+2 \theta+ \\
& \delta+\tau(\beta \alpha+\lambda \eta+\psi \phi+\sigma \chi)<1 .
\end{aligned}
$$

Then there exist $x \in B, u \in \widetilde{P}(x), v \in \widetilde{Q}(x), w \in \widetilde{R}(x)$ and $r \in \widetilde{S}(x)$ such that $(x, u, v, w, r)$ is a solution of (CGQVIP) and the sequences $\left\{x_{n}\right\},\left\{u_{n}\right\},\left\{v_{n}\right\},\left\{w_{n}\right\}$ and $\left\{r_{n}\right\}$ generated by Algorithm 3.1 converge strongly to $x, u, v, w$ and $r$, re- 
spectively, that is, $x_{n} \rightarrow x, u_{n} \rightarrow u, v_{n} \rightarrow v, w_{n} \rightarrow w$ and $r_{n} \rightarrow r$ as $n \rightarrow \infty$

Proof. By (1), we have

$$
\begin{aligned}
& \left\|x_{n+1}-x_{n}\right\|=\| x_{n}-G\left(x_{n}\right)+m\left(x_{n}\right)+Q_{X}\left[G\left(x_{n}\right)-\tau\left(T\left(u_{n}\right)\right.\right. \\
& \left.\left.+A\left(v_{n}\right)+M\left(w_{n}\right)+N\left(r_{n}\right)\right)-m\left(x_{n}\right)\right]-\left(x_{n-1}-G\left(x_{n-1}\right)\right. \\
& +m\left(x_{n-1}\right)+Q_{X}\left[G\left(x_{n-1}\right)-\tau\left(T\left(u_{n-1}\right)+A\left(v_{n-1}\right)\right.\right. \\
& \left.\left.+M\left(w_{n-1}\right)+N\left(r_{n-1}\right)\right)-m\left(x_{n-1}\right)\right] \| \\
& \leq\left\|x_{n}-x_{n-1}-\left(G\left(x_{n}\right)-G\left(x_{n-1}\right)\right)\right\|+2\left\|m\left(x_{n}\right)-m\left(x_{n-1}\right)\right\| \\
& +\left\|G\left(x_{n}\right)-G\left(x_{n-1}\right)\right\|+\tau\left\|T\left(u_{n}\right)-T\left(u_{n-1}\right)\right\| \\
& +\tau\left\|A\left(v_{n}\right)-A\left(v_{n-1}\right)\right\|+\tau\left\|M\left(w_{n}\right)-M\left(w_{n-1}\right)\right\| \\
& +\tau\left\|N\left(r_{n}\right)-N\left(r_{n-1}\right)\right\|
\end{aligned}
$$

From the proof of Theorem 3 in [4], we have

$$
\left\|x_{n}-x_{n-1}-\left(G\left(x_{n}\right)-G\left(x_{n-1}\right)\right)\right\|^{2} \leq\left(1-2 \gamma+64 D \delta^{2}\right)\left\|x_{n}-x_{n-1}\right\|^{2}
$$

It follows from the Lipschitz property of the corresponding functions that

$$
\begin{gathered}
\left\|m\left(x_{n}\right)-m\left(x_{n-1}\right)\right\| \leq \theta\left\|x_{n}-x_{n-1}\right\| \\
\left\|T\left(u_{n}\right)-T\left(u_{n-1}\right)\right\| \leq \alpha \beta\left\|x_{n}-x_{n-1}\right\| \\
\left\|A\left(v_{n}\right)-A\left(v_{n-1}\right)\right\| \leq \lambda \eta\left\|x_{n}-x_{n-1}\right\| \\
\left\|M\left(w_{n}\right)-M\left(w_{n-1}\right)\right\| \leq \psi \theta\left\|x_{n}-x_{n-1}\right\| \\
\left\|N\left(r_{n}\right)-N\left(r_{n-1}\right)\right\| \leq \sigma \chi\left\|x_{n}-x_{n-1}\right\| \\
\left\|G\left(x_{n}\right)-G\left(x_{n-1}\right)\right\| \leq \delta\left\|x_{n}-x_{n-1}\right\|
\end{gathered}
$$

From (3)-(10), we have

$$
\left\|x_{n+1}-x_{n}\right\| \leq t\left\|x_{n}-x_{n-1}\right\|,
$$

Where

$$
t=\left(1-2 \gamma+64 D \delta^{2}\right)+2 \theta+\delta+\tau(\beta \alpha+\lambda \eta+\psi \varphi+\sigma \chi)
$$

and $0<t<1$ by (2). Consequently $\left\{x_{n}\right\}$ is a Cauchy sequence, and thus it converges to some $x \in B$. By (1), we have

$$
\begin{gathered}
\left\|u_{n}-u_{n-1}\right\| \leq \widetilde{H}\left(\widetilde{P}\left(x_{n}\right), \widetilde{P}\left(x_{n-1}\right)\right) \leq \beta\left\|x_{n}-x_{n-1}\right\| ; \\
\left\|v_{n}-v_{n-1}\right\| \leq \widetilde{H}\left(\widetilde{Q}\left(x_{n}\right), \widetilde{Q}\left(x_{n-1}\right)\right) \leq \eta\left\|x_{n}-x_{n-1}\right\| ; \\
\left\|w_{n}-w_{n-1}\right\| \leq \widetilde{H}\left(\widetilde{R}\left(x_{n}\right), \widetilde{R}\left(x_{n-1}\right)\right) \leq \psi\left\|x_{n}-x_{n-1}\right\| ; \\
\left\|r_{n}-r_{n-1}\right\| \leq \widetilde{H}\left(\widetilde{S}\left(x_{n}\right), \widetilde{S}\left(x_{n-1}\right)\right) \leq \sigma\left\|x_{n}-x_{n-1}\right\|
\end{gathered}
$$

and hence $\left\{u_{n}\right\},\left\{v_{n}\right\},\left\{w_{n}\right\}$ and $\left\{r_{n}\right\}$ are also Cauchy sequences in $B$. Let $\left\{u_{n}\right\},\left\{v_{n}\right\},\left\{w_{n}\right\}$ and $\left\{r_{n}\right\}$ converge to some $u \in B, v \in B, w \in B, r \in B$ respectively. Since
$Q_{X}, G, \widetilde{P}, \widetilde{Q}, \widetilde{R}, T, A, M, N$ and $m$ are all continuous, we have

$$
\begin{aligned}
& x=x-G(x)+m(x)+Q_{X}[G(x)-\tau(T(u)+A(v) \\
& +M(w)+N(r))-m(x)] .
\end{aligned}
$$

Further, we have

$$
\begin{aligned}
& d(u, \tilde{P}(x))=\inf \{\|u-z\|: z \in \tilde{P}(x)\} \\
& \leq\left\|u-u_{n}\right\|+d\left(u_{n}, \tilde{P}(x)\right) \\
& \leq\left\|u-u_{n}\right\|+\tilde{H}\left(\tilde{P}\left(x_{n}\right), \tilde{P}(x)\right) \\
& \leq\left\|u-u_{n}\right\|+\beta\left\|x_{n}-x\right\| \rightarrow 0
\end{aligned}
$$

As

$$
n \rightarrow \infty
$$

and hence $u \in \widetilde{P}(x)$. Similarly we can show that $v \in \widetilde{Q}(x)$, $w \in \widetilde{R}(x)$ and $r \in \widetilde{S}(x)$. The result then follows from Lemma 3.2 .

\section{References}

[1] R. Ahmad and A. P. Farajzadeh, "On random variational inclusions with random fuzzy mappings and random relaxed cocoercive mappings," Fuzzy Sets \& Systems, vol. 160(21), 2009, pp. 3166-3174.

[2] R. Ahmad, F. Usman and S. S. Irfan, "Mixed variational inclusions and $T$-resolvent equations with fuzzy mappings," J. Fuzzy Mathematics, vol. 17(2), 2009, pp. 437-450.

[3] Ya. Alber, "Metric and generalized projection operators in Banach spaces: properties and applications," in Theory and Applications of Nonlinear Operators of Monotone and Accretive Type, A. Kartsatos, Ed. New York: Marcel Dekkar, 1996, pp. 15-50.

[4] Ya. Alber and J. C. Yao, "Algorithm for grnrralized multivalued co-variational inequalities in Banach spaces," Functional Diff. Equ., vol. 7, 2000, pp.5-13.

[5] Banyamini, J. Lindenstrauss, Geometric Nonlinear Functional Analysis I, AMS, colloquium Publications, vol. 48, 2000 .

[6] S. S. Chang, "Coincidence theorem and variational inequalities for fuzzy mappings," Fuzzy Sets and Systems, vol. 61, 1994, pp. 359-368.

[7] S. S. Chang and N. J. Huang, "Generalized complementarity problems for fuzzy mappings," Fuzzy Sets and Systems, vol. 55,1993 , pp. 227-234.

[8] S. S. Chang, Y. G. Zhu, "On variational inequalities for fuzzy mappings," Fuzzy Sets and Systems, vol. 32, 1989, pp. 359-367.

[9] K. Goebel and S. Reich, Uniform Convexity, Hyperbolic Geometry and Nonexpansive Mappings, New York: Marcel Dekker Inc., 1984.

[10] M. F. Khan and Salahuddin, "Generalized cocomplementarity problems in p-uniformly smooth Banach spaces,” J. Inequal. Pure \& Appl. Math., vol. 7(2), 2006, pp. $1-11$. 
[11] G. M. Lee, D. S. Kim, B. S. Lee and S. J. Cho, "Generalized vector variational inequality and fuzzy extension," Appl. Math. Lett., vol. 66, 1993, pp. 47-51.

[12] S. B. Nadler Jr., "Multi-valued contraction mappings," Pacific J. Math., vol. 30, 1969, pp.475-488.

[13] M. A. Noor, "Variational inequalities for fuzzy mappings
(I),” Fuzzy Sets and Systems, vol. 56, 1993, pp. 309-312.

[14] A. H. Siddiqi and S. S. Irfan, "Completely generalized cocomplementarity problems involving $p$-relaxed accretive operators with fuzzy mappings, in Nonlinear Analysis and Variational Problems, Pardalos, Rassias and A. Khan Ed., New York: Springer-Verlag, 2009, pp. 451-462. 